\title{
Angle-Resolved Photoemission Spectroscopy: Momentum-Space Microscope
}

\author{
Chan-Cuk Hwang* \\ Beamline Research Division, Pohang Accelerator Laboratory, Pohang University of Science and Technology, Pohang 37673, Korea
}

*Correspondence to:

Hwang CC,

Tel: +82-54-279-1254

Fax: +82-54-279-1599

E-mail: cchwang@postech.ac.kr

Received September 10, 2015

Revised September 13, 2015

Accepted September 13, 2015
In this review paper, I'd like to introduce the basics of angle-resolved photoemission spectroscopy (ARPES) and some of my results taken at the Pohang Accelerator Laboratory (PAL), the only synchrotron radiation in South Korea. The results show that ARPES is very useful, in particular, for studying two-dimensional materials. It looks like a microscope in momentum space similar to transmission electron microscope imaging atoms in real space.

Key Words: Angle-resolved photoemission spectroscopy, Pohang Accelerator Laboratory, Two-dimensional material, Momentum space

\section{INTRODUCTION}

Two-dimensional (2D) materials are a sort of solid consisting of surface only. Plenty of atoms and electrons in them are involved in determining their peculiar properties. Atoms and core electrons are localized, whereas valence electrons are delocalized in real space. Notice that most important properties, for example, transport, optical, magnetic ones, are determined by the delocalized valence electrons. How can we obtain information about the delocalized electrons? Fortunately, they are localized, whereas atoms are delocalized in momentum space. So if we know the structure of valence electrons in momentum space, we could understand where the important properties come from. Angle-resolved photoemission spectroscopy (ARPES) is the only tool that can see directly the electronic structure in momentum space (Hansson \& Uhrberg, 1988). ARPES is a kind of microscope imaging the electronic structure in momentum space. It resembles transmission electron microscope, a representative microscope that can image atoms. Since it is basically so sensitive to surface itself that it can be a useful tool to study 2D layered materials (Hüfner, 1995).

If light is incident on a solid, photoelectrons can be kicked out. If we measure the incident photon energy, work-function, electron's kinetic energy, then we can know the binding energy that holds electrons in their proper orbits within atoms inside materials from the Einstein's photoelectric effect (energy conservation) (Einstein, 1905).

$$
\mathrm{E}_{\mathrm{B}}=\hbar \omega-\mathrm{E}_{\mathrm{kin}}-\Phi
$$

$\left(E_{B}\right.$, binding energy of the electron; $\hbar \omega$, incoming photon energy; $E_{\text {kin }}$, kinetic energy of the outgoing electron; $\Phi$, electron work-function)

In addition, translational symmetry requires that the component of electron momentum parallel to the surface be conserved (momentum conservation). That is to say, an electron's momentum inside the crystal becomes its true momentum after it leaves. So, we can infer the true momentum inside the sample from the following equation for the momentum conservation by measuring the kinetic energy and the emission angle.

$$
\mathrm{k}_{\mathrm{i} \|}=\mathrm{k}_{\mathrm{fl}}=\sin \theta \sqrt{2 \mathrm{mE}_{\mathrm{kin}} / \hbar}
$$

$\left(\mathrm{k}_{\mathrm{i} \mid}\right.$, initial in-plane momentum of the electron; $\mathrm{k}_{\mathrm{f} \mid}$, in-plane momentum of the outgoing electron)

Both energy and momentum conservation allow us to determine the energy-momentum relation that decides most material's properties (Damascelli et al., 2003).

In this paper, I would like to introduce some of my results on graphene reported recently using ARPES at the 10D beamline

This work was supported by the National Research Foundation of Korea (NRF) through the SRC Center for Topological Matter (No. 2011-0030787) and the Basic Science Research Program (No. 2011-0009427). The experiments at PLS were supported in part by MSIP and POSTECH.

(a) This is an open-access article distributed under the terms of the Creative Commons Attribution Non-Commercial License (http://creativecommons.org/licenses/by-nc/4.0) which permits unrestricted noncommercial use, distribution, and reproduction in any medium, provided the original work is properly cited.

Copyrights @ 2015 by Korean Society of Microscopy 
of the Pohang Accelerator Laboratory (PAL) and provide insight into how ARPES can be utilized in the field of layered materials.

\section{RESULTS AND DISCUSSION}

Graphene, an emergent 2D carbon sheet, has extraordinary electrical properties due to the linear energy band dispersion at the Dirac point. Charge carriers in graphene behave like relativistic particles with zero rest mass, resulting in ballistic transport on the micrometer scale at room temperature. This characteristic makes graphene a promising material for future electronic devices, and enormous progress has been made since the existence of graphene was clearly demonstrated in 2004 (Novoselov et al., 2004).

The synthesis of a realistic monolayer graphene via a selflimiting process is achieved on $\mathrm{Cu}$ foil as a result of the low carbon solubility at growth temperatures. Thus, $\mathrm{Cu}$ is considered to be the most promising substrate for the growth of high-quality and large area graphene by chemical vapor deposition (CVD), in particular, on the (111) facet. Because the interactions between graphene and $\mathrm{Cu}$ substrates influence the orientation, quality, and properties of the synthesized graphene, the interactions using ARPES is investigated (Jeon et al., 2013).

The evolution of both the Shockley surface state of the $\mathrm{Cu}(111)$ and the $\pi$ band of the graphene is measured from the initial stage of CVD growth to the formation of a monolayer, as shown in Fig. 1. Graphene growth is initiated along the $\mathrm{Cu}(111)$ lattice, where the Dirac band crossed the Fermi energy $\left(E_{\mathrm{F}}\right)$ at the $K$ point without hybridization with the d-band of $\mathrm{Cu}$. Then two rotated domains are additionally grown as the area covered with graphene becomes wider. The Dirac energy is about $-0.4 \mathrm{eV}$ and the energy of the Shockley surface state of $\mathrm{Cu}(111)$ shifts toward the $E_{\mathrm{F}}$ by $\sim 0.15 \mathrm{eV}$ upon graphene formation. These results indicate weak interactions between graphene and $\mathrm{Cu}$, and that the electron transfer is limited to that between the Shockley surface state of $\mathrm{Cu}(111)$ and the $\pi$ band of graphene. This weak interaction and slight lattice mismatch between graphene and $\mathrm{Cu}$ result in the growth of rotated graphene domains $\left(9.6^{\circ}\right.$ and $\left.8.4^{\circ}\right)$, which show no significant differences in the Dirac band with respect to different orientations. These rotated graphene domains result in grain boundaries which would hinder a large-sized single monolayer growth on $\mathrm{Cu}$ substrates.

On the other hand, since the lattice of graphene is well matched to that of $\mathrm{Ni}$, graphene grown on a single-crystal $\mathrm{Ni}(111)$ surface does not show different in-plane orientations. However, the unique electronic structure of graphene exhibiting linear dispersion disappears due to the strong interaction of graphene with the Ni substrate. To restore the pristine electronic structure of graphene, it is essential to remove the substrate with acids and then to transfer graphene to weakly interacting substrates. However, removing the metal substrate from a graphene/metal system and transferring the graphene to other substrates is not an easy task, and these processes can cause undesirable tears, defects, and impurities in graphene.

In Park et al. (2014), we show that $\mathrm{Na}$ can be used to restore the electronic structure of graphene from a strongly interacting $\mathrm{Ni}(111)$ surface that is characterized by using densityfunctional-theory (DFT) calculations and ARPES.

We carry out DFT calculations by employing the Vienna ab initio simulation package with the use of the spin-polarized local density approximation and the projector-augmentedwave method with a plane-wave basis set of $400 \mathrm{eV}$. For the graphene/ $\mathrm{Ni}(111)$ surface, the $\mathrm{C}$ atoms of graphene are aligned on the top and fcc sites of $\mathrm{Ni}(111)$. We have considered both (adsorption and intercalation) possibilities for given $\mathrm{Na}$ coverages. Except for the low coverage of $0.16 \mathrm{ML}$, $\mathrm{Na}$ intercalation is favored over surface adsorption with more
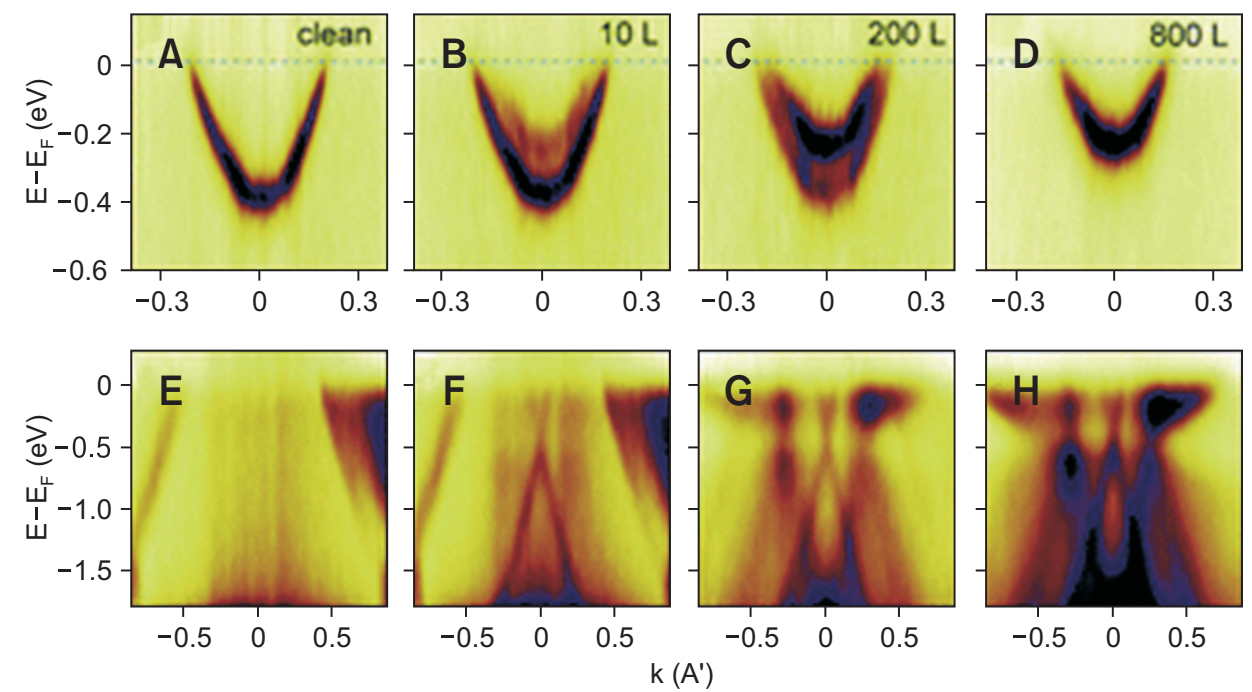

Fig. 1. The evolution of the Shockley surface state of $\mathrm{Cu}(111)$ (A-D) and the Dirac bands of graphene (E-H) with increasing $\mathrm{C}_{2} \mathrm{H}_{2}$ dose from 0 to $800 \mathrm{~L}$. 
energy gains of about $1 \mathrm{eV}$. In all $\mathrm{Na}$ coverages considered, we find that the graphene $\pi$ bands form a well-defined Dirac point. It is notable that a small intercalation of $0.16 \mathrm{ML}$ is enough to recover the Dirac-cone shape of ideal graphene. The Dirac point shifts down with coverage up to $0.63 \mathrm{ML}$.

Fig. 2 shows the majority-spin band structures of the graphene/ $\mathrm{Ni}(111)$ surface before and after $0.63-\mathrm{ML} \mathrm{Na}$ deposition. In Fig. 2A, the important feature of the graphene/ $\mathrm{Ni}(111)$ band structure is that the Dirac-cone shape of graphene $\pi$ bands is significantly disturbed. Interestingly, in Fig. 2B, the adsorbed $\mathrm{Na}$ atoms little affect the electronic structure of graphene/Ni(111). In contrast, as seen in Fig. 2C, Na intercalation perfectly recovers the Dirac-cone shape of ideal graphene. We find that, due to the interaction with $\mathrm{Na}$, the graphene bands shift downward by approximately $1.2 \mathrm{eV}$ from the Fermi level, indicating a large charge transfer from the $\mathrm{Na} / \mathrm{Ni}(111)$ surface to the graphene layer. While the $\mathrm{Na}$ atoms interact strongly with the $\mathrm{Ni}$ substrate, the $\mathrm{Na}-\mathrm{Na}$ interaction is so weak that the Na-derived states are empty at approximately $3 \mathrm{eV}$ above the Fermi level.

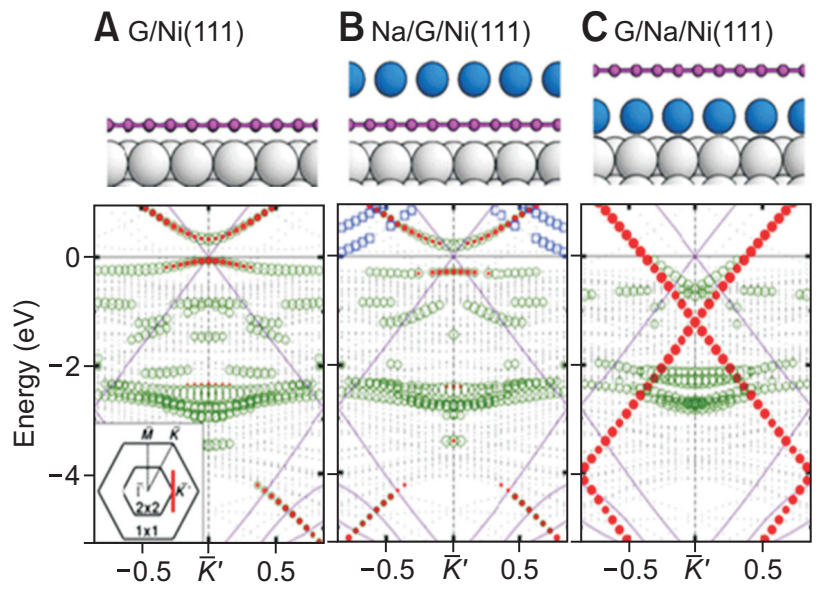

Fig. 2. Majority-spin band structures of graphene/Ni(111) (A), Na/ graphene $/ \mathrm{Ni}(111)(B)$, and graphene/Na/Ni(111) (C). The filled red (open green) circles represent $\mathrm{C}$-derived (Ni-derived) surface states that contain more than $20 \%$ ( $40 \%$ ) of charge in the graphene layer (topmost Ni layer).
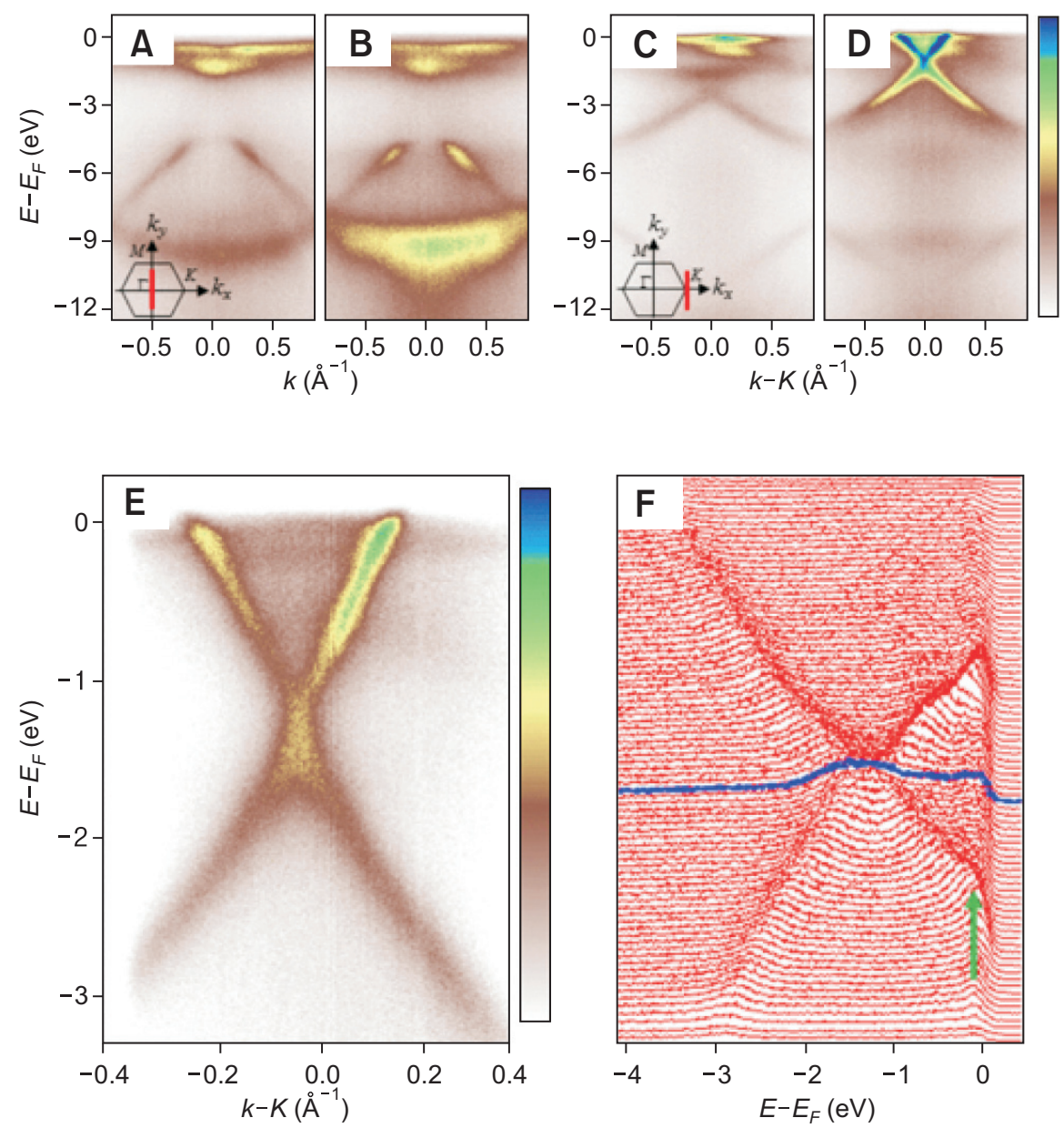

Fig. 3. Electronic band structures measured by angle-resolved photoemission spectroscopy of the as-grown graphene (A, C) and Na-intercalated graphene (B, D), and high angular and energyresolution measurements of the $\mathrm{Na}$ intercalated graphene (E, F). (A, B) Taken at the $\Gamma$ point; $(\mathrm{C}, \mathrm{D})$ taken at the K point. (E) Band structure around the $\mathrm{K}$ point near the Fermi energy, taken along the direction perpendicular to the $\Gamma-\mathrm{K}$. (F) Photoemission intensity profiles as a function of binding energy. 
Fig. 3 shows the electronic band structure measured by ARPES of the as-grown graphene on $\mathrm{Ni}(111)$ and the graphene/ $\mathrm{Ni}(111)$ intercalated with $\mathrm{Na}$ near the $\Gamma$ and $\mathrm{K}$ points. By inspecting the energy band dispersions of the as-grown graphene/Ni(111) sample shown in Fig. 3A and C, the binding energies of the $\pi$ state are observed to be about -10 and -2.7 $\mathrm{eV}$ at the $\Gamma$ and $\mathrm{K}$ points, respectively. This can be explained by strong hybridization of the $\pi$ orbitals of graphene and the d orbitals of the $\mathrm{Ni}(111)$ metal surface. Fig. 3B and D show the electronic band structures after $\mathrm{Na}$ intercalation near the $\Gamma$ and $\mathrm{K}$ points, respectively. While the $\sigma, \pi$, and $\mathrm{Ni} 3 \mathrm{~d}$ bands are not considerably changed near the $\Gamma$ point, a characteristic $\pi$ band appears near the $\mathrm{K}$ point. The introduction of $\mathrm{Na}$ atoms to the interface between the graphene and the $\mathrm{Ni}(111)$ surface weakens the strong hybridization between $\mathrm{C} 2 \mathrm{p}$ and $\mathrm{Ni} 3 \mathrm{~d}$ orbitals, causing the increase of distance between the graphene and the metal substrate. Because the graphene layer detaches from the $\mathrm{Ni}(111)$ surface, the linear band dispersion is completely recovered. According to our DFT calculations, a simple adsorption of $\mathrm{Na}$ atoms on top of the graphene/Ni(111) system does not recover the Diraccone band structure of graphene. Unlike $\mathrm{Au}, \mathrm{Al}$, etc., $\mathrm{Na}$ atoms adsorbed on the graphene surface penetrate into the graphene spontaneously at room temperature. This seems to be related closely with the tiny diffusion barrier for $\mathrm{Na}$ atoms. The spontaneous intercalation makes the graphene layer nearly electronically ideal, thereby showing its inherent sixfold symmetry in scanning tunneling microscopy and characteristic bands featuring the Dirac-cone structure with a negligible band gap. The intercalated $\mathrm{Na}$ atoms could be easily oxidized to become an insulator. Thus, it provides new possibilities toward the high-quality, large-area, and quasifree-standing graphene on metal or insulating substrates without etching and transferring processes.

\section{SUMIMARY}

The two examples show how ARPES is useful in the field of $2 \mathrm{D}$ materials like graphene. It is noted that a large number of papers on other layered materials are being published. ARPES looks like a momentum-space microscope, revealing the structure of valence electrons in momentum space. It gives much more information about transport, optical, magnetic properties, etc. Besides, details of the electronic structure are considerably related to intriguing quasiparticle interactions, although the analysis is not so simple.

\section{CONFLICT OF INTEREST}

No potential conflict of interest relevant to this article was reported.

\section{REFERENCES}

Damascelli A, Hussain Z, and Shen Z X (2003) Angle-resolved photoemission studies of the cuprate superconductors. Reviews of Modern Physics 75, 473.

Einstein A (1905) Concerning an Heuristic point of view toward the emission and transformation of light. Ann. Phys. 17, 132.

Hansson G V and Uhrberg R I G (1988) Photoelectron spectroscopy of surface states on semiconductor surfaces. Surface Science Reports 9. 197-292.

Hüfner S (1995) Photoelectron Spectroscopy: Principles and Applications (Springer, Berlin)
Jeon C, Hwang H N, Lee W G, Jung Y G, Kim K S, Park C Y, and Hwang C C (2013) Rotated domains in chemical vapor deposition-grown monolayer graphene on $\mathrm{Cu}(111)$ : an angle-resolved photoemission study. Nanoscale 5, 8210.

Novoselov K S, Geim A K, Morozov S V, Jiang D, Zhang Y, Dubonos S V, Grigorieva I V, and Firsov A A (2004) Electric field effect in atomically thin carbon films. Science 306, 666 .

Park Y S, Park J H, Hwang H N, Laishram T S, Kim K S, Kang M H, and Hwang C C (2014) Quasi-free standing graphene monolayer on a $\mathrm{Ni}$ crystal through spontaneous $\mathrm{Na}$ intercalation. Phys. Rev. $X \mathbf{4}$, 031016. 